\title{
Correction to: Prognostic Role of Blood Eosinophil Count in Patients with Sorafenib-Treated Hepatocellular Carcinoma
}

\author{
Giulia Orsi ${ }^{1}$. Francesco Tovoli ${ }^{2,3}$. Vincenzo Dadduzio ${ }^{4}$ Caterina Vivaldi ${ }^{5,6}$. Oronzo Brunetti ${ }^{7}$. Luca lelasi ${ }^{2,3}$. \\ Fabio Conti ${ }^{8}$. Giulia Rovesti ${ }^{1}$. Laura Gramantieri ${ }^{9} \cdot$ Mario Domenico Rizzato ${ }^{4,10} \cdot$ Irene Pecora $^{5}$. \\ Antonella Argentiero $^{7}$. Federica Teglia ${ }^{2,3}$. Sara Lonardi ${ }^{4}$. Francesca Salani ${ }^{5}$. Alessandro Granito ${ }^{2,3}$. \\ Vittorina Zagonel ${ }^{4}$. Giorgia Marisi ${ }^{11}$. Giuseppe Cabibbo ${ }^{12}$. Francesco Giuseppe Foschi ${ }^{8}$. Francesca Benevento ${ }^{2}$. \\ Alessandro Cucchetti ${ }^{2}$. Fabio Piscaglia ${ }^{2,3} \cdot$ Stefano Cascinu ${ }^{13,14} \cdot$ Mario Scartozzi $^{15}$ - Andrea Casadei-Gardini ${ }^{13,14}$
}

Published online: 28 October 2020

(c) Springer Nature Switzerland AG 2020

\section{Correction to: Targeted Oncology} https://doi.org/10.1007/s11523-020-00757-3

An Online First version of this article was made available online at https://link.springer.com/article/10.1007/s1152 3-020-00757-3 on 12 October 2020. Errors were subsequently identified in the article, and the following corrections should be noted.

Page 1 and 13: The listing of the author names and affiliations, which previously read:

"Giulia Orsi ${ }^{1}$, Francesco Tovoli ${ }^{2,3}$, Vincenzo Dadduzio ${ }^{4}$, Caterina Vivaldi ${ }^{5,6}$, Oronzo Brunetti ${ }^{7}$, Luca Ielasi ${ }^{2}$, Fabio

The original article can be found online at https://doi.org/10.1007/ s11523-020-00757-3.

Andrea Casadei-Gardini

casadeigardini@gmail.com

1 Department of Oncology and Hematology, Division of Oncology, University Hospital of Modena, Via del Pozzo 71, Modena, Italy

2 Department of Medical and Surgical Sciences-DIMEC, Alma Mater Studiorum-University of Bologna, Bologna, Italy

3 Division of Internal Medicine, Azienda Ospedaliero-Universitaria di Bologna, via Albertoni 15, Bologna, Italy

4 Medical Oncology Unit 1, Department of Clinical and Experimental Oncology, Istituto Oncologico Veneto, IRCCS, Padua, Italy

5 Division of Medical Oncology 2, Pisa University Hospital, Pisa, Italy

6 Department of Translational Research and New Surgical and Medical Technologies, University of Pisa, Via Savi 6, 56126 Pisa, Italy
Conti $^{8}$, Giulia Rovesti ${ }^{1}$, Laura Gramantieri ${ }^{9}$, Mario Domenico Rizzato ${ }^{4,10}$, Irene Pecora ${ }^{5}$, Antonella Argentiero ${ }^{7}$, Federica Teglia ${ }^{2}$, Sara Lonardi ${ }^{2}$, Francesca Salani ${ }^{5}$, Alessandro Granito ${ }^{2}$, Vittorina Zagonel ${ }^{4}$, Giorgia Marisi ${ }^{11}$, Giuseppe Cabibbo ${ }^{12}$, Francesco Giuseppe Foschi ${ }^{8}$, Francesca Benevento ${ }^{2}$, Alessandro Cucchetti ${ }^{2}$, Fabio Piscaglia $^{2}$, Stefano Cascinu ${ }^{13,14}$, Mario Scartozzi ${ }^{15}$, Andrea Casadei-Gardini ${ }^{13,14}$

${ }^{1}$ Department of Oncology and Hematology, Division of Oncology, University Hospital of Modena, Via del Pozzo 71, Modena, Italy

${ }^{2}$ Department of Medical and Surgical Sciences - DIMEC, Alma Mater Studiorum-University of Bologna, Bologna, Italy

Medical Oncology Unit, IRCCS Istituto Tumori "Giovanni Paolo II", Bari, Italy

8 Department of Internal Medicine, Faenza Hospital, AUSL Romagna, Faenza, Italy

9 Center for Applied Biomedical Research (CRBa), St. Orsola-Malpighi University Hospital, Bologna, Italy

10 Department of Surgery, Oncology and Gastroenterology, University of Padova, Padua, Italy

11 Biosciences Laboratory, Istituto Scientifco Romagnolo per lo Studio e la Cura dei Tumori (IRST), IRCCS, Meldola, Italy

12 Section of Gastroenterology and Hepatology, PROMISE, University of Palermo, Palermo, Italy

13 Vita-Salute San Rafaele University, Milan, Italy

14 Department of Medical Oncology, San Rafaele Scientifc Institute IRCCS, Milan, Italy

15 Department of Medical Oncology, University Hospital of Cagliari, Cagliari, Italy 
${ }^{3}$ Division of Internal Medicine, Azienda OspedalieroUniversitaria di Bologna, via Albertoni 15, Bologna, Italy

${ }^{4}$ Medical Oncology Unit 1, Department of Clinical and Experimental Oncology, Istituto Oncologico Veneto, IRCCS, Padua, Italy

${ }^{5}$ Division of Medical Oncology 2, Pisa University Hospital, Pisa, Italy

${ }^{6}$ Department of Translational Research and New Surgical and Medical Technologies, University of Pisa, Via Savi 6, Pisa 56126, Italy

${ }^{7}$ Medical Oncology Unit, IRCCS Istituto Tumori "Giovanni Paolo II", Bari, Italy

${ }^{8}$ Department of Internal Medicine, Faenza Hospital, AUSL Romagna, Faenza, Italy

${ }^{9}$ Center for Applied Biomedical Research (CRBa), St. Orsola-Malpighi University Hospital, Bologna, Italy

${ }^{10}$ Department of Surgery, Oncology and Gastroenterology, University of Padova, Padua, Italy

${ }^{11}$ Biosciences Laboratory, Istituto Scientifco Romagnolo per lo Studio e la Cura dei Tumori (IRST), IRCCS, Meldola, Italy

${ }^{12}$ Section of Gastroenterology and Hepatology, PROMISE, University of Palermo, Palermo, Italy

${ }^{13}$ Vita-Salute San Rafaele University, Milan, Italy

${ }^{14}$ Department of Medical Oncology, San Rafaele Scientifc Institute IRCCS, Milan, Italy

${ }^{15}$ Department of Medical Oncology, University Hospital of Cagliari, Cagliari, Italy"

Should read:

"Giulia Orsi ${ }^{1}$, Francesco Tovoli ${ }^{2,3}$, Vincenzo Dadduzio ${ }^{4}$, Caterina Vivaldi ${ }^{5,6}$, Oronzo Brunetti ${ }^{7}$, Luca Ielasi ${ }^{2,3}$, Fabio Conti $^{8}$, Giulia Rovesti ${ }^{1}$, Laura Gramantieri ${ }^{9}$, Mario Domenico Rizzato ${ }^{4,10}$, Irene Pecora ${ }^{5}$, Antonella Argentiero ${ }^{7}$, Federica Teglia ${ }^{2,3}$, Sara Lonardi ${ }^{4}$, Francesca Salani $^{5}$, Alessandro Granito ${ }^{2,3}$, Vittorina Zagonel ${ }^{4}$, Giorgia Marisi ${ }^{11}$, Giuseppe Cabibbo ${ }^{12}$, Francesco Giuseppe Foschi $^{8}$, Francesca Benevento ${ }^{2}$, Alessandro Cucchetti ${ }^{2}$,
Fabio Piscaglia ${ }^{2,3}$, Stefano Cascinu ${ }^{13,14}$, Mario Scartozzi ${ }^{15}$, Andrea Casadei-Gardini ${ }^{13,14}$

${ }^{1}$ Department of Oncology and Hematology, Division of Oncology, University Hospital of Modena, Via del Pozzo 71, Modena, Italy

${ }^{2}$ Department of Medical and Surgical Sciences-DIMEC, Alma Mater Studiorum-University of Bologna, Bologna, Italy

${ }^{3}$ Division of Internal Medicine, Azienda OspedalieroUniversitaria di Bologna, via Albertoni 15, Bologna, Italy

${ }^{4}$ Medical Oncology Unit 1, Department of Clinical and Experimental Oncology, Istituto Oncologico Veneto, IRCCS, Padua, Italy

${ }^{5}$ Division of Medical Oncology 2, Pisa University Hospital, Pisa, Italy

${ }^{6}$ Department of Translational Research and New Surgical and Medical Technologies, University of Pisa, Via Savi 6, Pisa 56126, Italy

${ }^{7}$ Medical Oncology Unit, IRCCS Istituto Tumori "Giovanni Paolo II", Bari, Italy

${ }^{8}$ Department of Internal Medicine, Faenza Hospital, AUSL Romagna, Faenza, Italy

${ }^{9}$ Center for Applied Biomedical Research (CRBa), St. Orsola-Malpighi University Hospital, Bologna, Italy

${ }^{10}$ Department of Surgery, Oncology and Gastroenterology, University of Padova, Padua, Italy

${ }^{11}$ Biosciences Laboratory, Istituto Scientifco Romagnolo per lo Studio e la Cura dei Tumori (IRST), IRCCS, Meldola, Italy

${ }^{12}$ Section of Gastroenterology and Hepatology, PROMISE, University of Palermo, Palermo, Italy

${ }^{13}$ Vita-Salute San Rafaele University, Milan, Italy

${ }^{14}$ Department of Medical Oncology, San Rafaele Scientifc Institute IRCCS, Milan, Italy

${ }^{15}$ Department of Medical Oncology, University Hospital of Cagliari, Cagliari, Italy"

The original article has been updated. 